\title{
Mosaic-Based 3D Scene Representation and Rendering
}

\author{
Zhigang Zhu \\ Department of Computer Science \\ City College of New York, New York, NY 10031 \\ zhu@cs.ccny.cuny.edu
}

\author{
Allen R. Hanson \\ Department of Computer Science \\ University of Massachusetts Amherst, MA 01003 \\ hanson@cs.umass.edu
}

\begin{abstract}
In this paper we address the problem of fusing images from many video cameras or a moving video camera. The captured images have obvious motion parallax, but they will be aligned and integrated into a few mosaics with a large field-ofview (FOV) that preserve 3D information. We have developed a geometric representation that can re-organize the original perspective images into a set of parallel projections with different oblique viewing angles. In addition to providing a wide field of view, mosaics with various oblique views well represent occlusion regions that cannot be seen in a usual nadir view. Stereo pair(s) can be formed from a pair of mosaics with different oblique viewing angles and thus image-based $3 \mathrm{D}$ viewing can be achieved. This representation can be used as both an advanced video interface and a pre-processing step for 3D reconstruction.
\end{abstract}

Keywords - stereo mosaics; image-based rendering; visual representation;

\section{INTRODUCTION}

A 2D panoramic mosaic of a 3D scene generated from video from a translating camera has the geometry of multiple viewpoints [1,2], but it only preserves information from a single viewing direction. Three-dimensional (3D) structure and surface information from other viewing directions of the original video is lost in such a representation. A digital elevation map (DEM) generated from aerial photometry consists of a sampled array of elevations (depths) for a number of ground positions at regularly spaced intervals [3]. It usually only has a nadir viewing direction, hence the surfaces from other viewing directions cannot be represented. However, in some applications such as surveillance and security inspection, a scene or an object (e.g. a vehicle) needs to be observed from many viewing directions to reveal anomalies hidden in unusual views. Stereo panoramas [4,5] have been presented as a mechanism for obtaining the "best" 3D information from an off-center rotating camera. In the case of a translating camera, various layered representations [6-8] have been proposed to represent both $3 \mathrm{D}$ information and occlusions, but such representations need 3D reconstructions.

This paper presents an approach for fusing images from many spatially distributed video cameras or a moving video camera into a few mosaiced images that preserve 3D information. In both cases, a virtual 2D array of cameras with FOV overlaps is formed to generate complete coverage of a scene (or an object). Note that many viewing directions are already included in the original camera views. This property has been used in the X-slit mosaics with non-parallel rays [9] for image-based rendering. In this paper we propose a representation that can re-organize the original perspective images into a set of parallel projections with different oblique viewing angles (in both the $x$ and the $y$ directions of the 2D images). Mosaics with 2D oblique parallel projections are a unified representation for our previous work on parallelperspective stereo mosaics $[11,12]$ and multi-camera mosaics $[13,14]$. Such representations provide a wide field of view, optimal 3D information for stereo viewing and reconstruction, and the capability to represent occlusions. This paper is organized as follows. In addition to the description and discussions of the unified presentation in Sections II and III, we also present a general ray interpolation method in Section IV, and a mosaic-based 3D rendering method in Section V. Experimental results are given in Section VI for two important applications - aerial video surveillance and under vehicle inspection. Section VII is a brief summary.

\section{2D OBliQue Parallel ProJeCtion}

A normal perspective camera has a single viewpoint, which means all the light rays pass through a common nodal point. On the other hand, an orthogonal image with parallel projections in both the $x$ and $y$ directions has all the rays parallel to each other. Imagining that we have a sensor with parallel projections, we could turn the sensor to capture images with different oblique angles (including both nadir and oblique angles) in both the $x$ and $y$ directions. Thus we can create many pairs of parallel stereo images, each with two different oblique angles, and observe surfaces occluded in a nadir view.

Fig. 1 shows the parallel stereo in a 1D case, where two oblique angles $\beta_{1}$ and $\beta_{2}$ are chosen. The depth of a point can be calculated as

$$
Z=\frac{B}{\tan \beta_{2}-\tan \beta_{1}}
$$

where $\beta_{1}$ is $\beta_{2}$ are the angles of the two viewing directions, respectively, and $B$ is the adaptive baseline between the two viewpoints that is proportional to the displacement of the corresponding points in the stereo mosaics. It has been shown by others [10] and by us [11, 12] that parallel stereo is 
superior to both conventional perspective stereo and to the recently developed multi-perspective stereo with concentric mosaics for 3D reconstruction (e.g., in [5]). The adaptive baseline inherent in the parallel-perspective geometry permits depth accuracy independent of absolute depth in theory $[10,11]$ and as a linear function of depth in stereo mosaics generated from perspective image sequences [12]. In contrast, the depth error of perspective stereo and the concentric stereo is proportional to the square of depth.

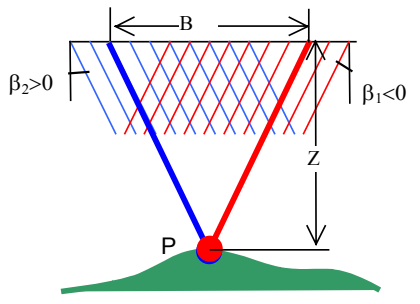

Fig. 1. Depth from parallel stereo with multiple viewpoints: 1D case.

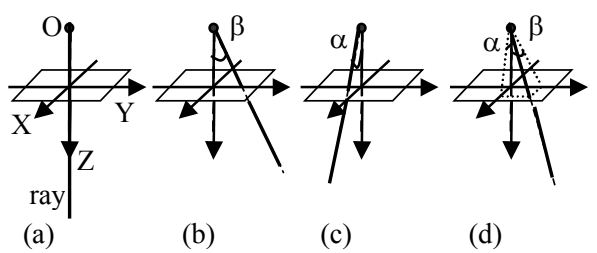

Fig. 2. Parallel projections with two oblique angles $\alpha$ and $\beta$ (in the $x$ and $y$ directions).

We can make two extensions to this parallel stereo. First, we can select various oblique angles for constructing multiple parallel projections. By doing so we can observe various degrees of occlusions and can construct stereo pairs with different depth resolution via the selection of different pairs of oblique angles. Second, we can extend this 1D parallel projection to $2 \mathrm{D}$ (Fig. 2): we can obtain a mosaiced image that has a nadir view (Fig. 2a), oblique angle(s) only in one direction (Fig. 2b and c) or in both the $x$ and the $y$ directions (Fig. 2d).

\section{2D (VIRTUAL) ARRAY OF CAMERAS}

It is impractical to use a single sensor to capture orthogonal images with full parallel projections in both $x$ and $y$ dimensions for a large scene, and with various oblique directions. However there are least three practical ways of generating images with oblique parallel projections using existing sensors: a 2D sensor array of many spatially distributed cameras (Fig. 3a), a "scanner" with a 1D array of cameras (Fig. 3b), and a single perspective camera that moves in $2 \mathrm{D}$ (Fig. $3 \mathrm{c}$ ).

With a 2D array of many perspective cameras (Fig. 3a), we first assume that the optical axes of all the cameras point in the same directions (into the paper in Fig 3a), and the viewpoints of all cameras are on a single plane perpendicular to their optical axes. Then we can reorganize the perspective images into mosaiced images with any oblique viewing angles by extracting rays from the original perspective images with the same viewing directions, one ray from each image. If the camera array is dense enough, then we can generate densely mosaiced images.

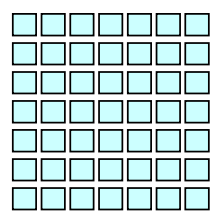

a

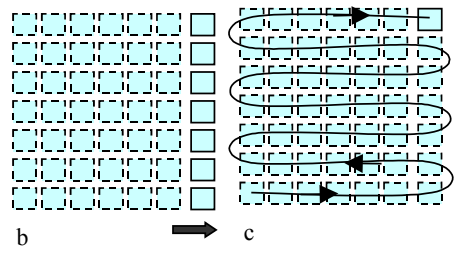

Fig. 3. Parallel mosaics from 2D bed of cameras. (a) 2D array; (b) 1D scan array and (c) a single scan camera.

If we only have a 1D linear array of perspective cameras (Fig. 3b), we can "scan" the camera array over the scene to synthesize a virtual 2D camera array. Then we can still generate stereo mosaic pairs with oblique parallel projections, given that we can accurately control the translation of the camera array. We have actually used this approach in an Under Vehicle Inspection System (UVIS) [13, 14, 18].

Even if we just use a single camera, we can still generate a $2 \mathrm{D}$ virtual bed of cameras by moving the camera in two dimensions, along a "2D scan" path shown in Fig. 3c. This is the case for aerial video mosaics $[11,12,15,17]$.

\section{PRISM: VIDEO MOSAICING AlgORITHM}

In real applications, there are two challenging issues. The first problem is camera orientation estimation (calibration). In our previous study on an aerial video application, we used external orientation instruments, i.e., GPS, INS and a laser profiler, to ease the problem of camera orientation estimation $[11,12]$. In this paper, we assume that the extrinsic and intrinsic camera parameters are known at each camera location. The second problem is to generate dense parallel mosaics with a sparse, uneven, camera array, and for a complicated 3D scene. To solve this problem, we have proposed a Parallel Ray Interpolation for Stereo Mosaics (PRISM) approach [11]. While the PRISM algorithm was originally designed to generate parallel-perspective stereo mosaics (parallel projection in one direction and perspective projection in the other), the core idea of ray interpolation can be used for generating a mosaic with full parallel projection at any oblique angle.

Fig. 4 shows how the PRISM works for 1D images. The 1D camera has two axes - the optical axis $(\mathrm{Z})$ and the $\mathrm{Y}$-axis. Given the known camera orientation at each camera location, one ray with a given oblique angle $\beta$ can be chosen from the image at each camera location to contribute to the parallel mosaic with this oblique angle $\beta$. The oblique angle is defined against the direction perpendicular to the mosaicing direction, which is the dominant direction of the camera path (Fig. 4). But the problem is that the "mosaiced" image with only those existing rays will be sparse and uneven since the camera array are usually not regular and very dense. Therefore interpolated parallel rays between a pair of existing parallel rays (from two neighboring images) are generated by performing local 
matching between these two images. The assumption is that we can find at least two images to generate the parallel ray. Such an interpolated ray is shown in Fig 4, where Ray I is interpolated from Image A and Image B.

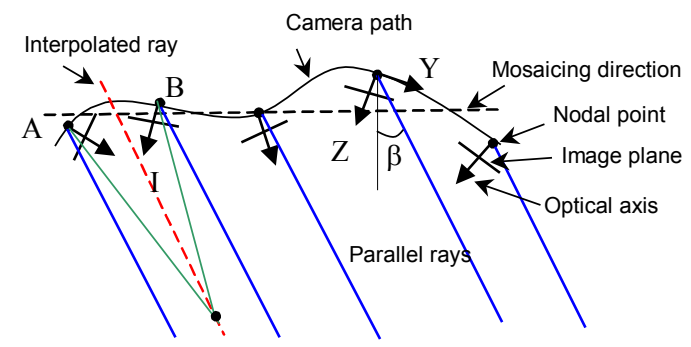

Fig. 4. Ray interpolation for parallel mosaicing from an camera array.

The extension of this approach to $2 \mathrm{D}$ images is straightforward, and a region triangulation strategy similar to that in [11] can be applied here to deal with 2D cases. One important issue here is the selection of neighborhood images for ray interpolation. For example, with a 1D scan sequence of a single camera, it is hard to generate full parallel projection in the $\mathrm{X}$ direction, which is perpendicular to the motion of the camera, since the interpolated parallel rays far off the center of the images in the $\mathrm{x}$ direction have to use rays with rather different oblique angles in the original perspective images.

\section{V.STEREO VIEWING AND 3D RECONSTRUCTION}

Parallel mosaics with various oblique angles represent scenes from the corresponding viewing angles with parallel rays and with wide fields of view. There are two obvious applications of such representation. First, a human can perceive the 3D scene from a pair of mosaics with different oblique angles (e.g. using polarized glasses) without any 3D recovery. If we have mosaics with various oblique angles in both the $\mathrm{x}$ and the $\mathrm{y}$ direction, we can generate a virtual fly/walk-through by noting that (1) the translation in the $x y$ plane can be simulated by shifting the current displayed mosaic pair, (2) the rotations around the $\mathrm{X}$ and the $\mathrm{Y}$ axes can be simulated by selecting different pairs of mosaics with different oblique angles, and (3) the rotation around the optical axis only needs to rotate the pair of mosaics in their image planes. The visual disparities can also be controlled by changing the angles between the two mosaics for stereo viewing.

Second, for 3D recovery, matches are only performed on a pair of mosaics, not on individual video frames. Stereo mosaic methods also solve the baseline versus field-of-view (FOV) dilemma efficiently by extending the FOV in the directions of mosaicing - in both the $x$ and $y$ directions. More importantly, the parallel stereo mosaics have optimal/adaptive baselines for all the points, which leads to uniform depth resolution in theory and linear depth resolution in practice. For 3D reconstruction, epipolar geometry is rather simple due to the full parallel projections in the mosaic pair.

\section{EXPERIMENTAL EXAMPLES}

\section{A. Video Mosaics from Aerial Video}

In theory, with a camera undergoing an ideal 1D translation and a nadir view direction, two spatio-temporal images can be generated by extracting two columns of pixels at the front and rear edges of each frame in motion (Fig. 5). The mosaic images thus generated are parallel-perspective, with parallel projection in the motion direction and perspective projection in the other. In our aerial video environmental monitoring application, a single camera is mounted in a small aircraft undergoing 6 DOF motion, together with a GPS, INS and laser profiler to measure the moving camera locations and the distance to the terrain $[11,12]$. So we generate seamless stereo parallel-perspective video mosaic strips from image sequences with a $1 \mathrm{D}$ scan path but with a rather general motion model, using the proposed parallel ray interpolation for stereo mosaicing (PRISM) approach [11]. In the PRISM approach for large-scale 3D scene modeling, the computation is efficiently distributed in three steps: camera pose estimation via the external measurement units, image mosaicing via ray interpolation, and $3 \mathrm{D}$ reconstruction from a pair of stereo mosaics $[11,12,15]$.

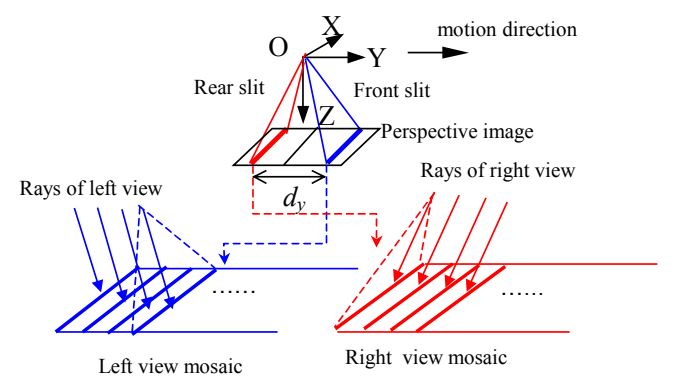

Fig. 5. Parallel-perspective stereo mosaics with a 1D camera scan path

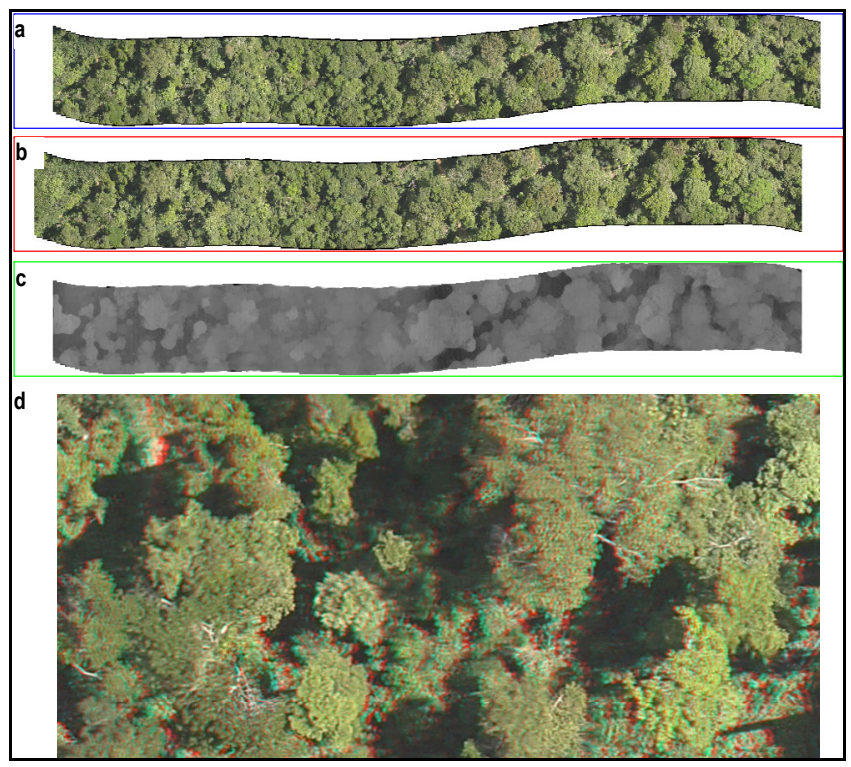

Fig. 6. Stereo mosaics and $3 \mathrm{D}$ reconstruction of a 166 -frame telephoto video sequence. 
Fig. 6 shows a real example of stereo mosaics (with two yoblique angles) generated from a telephoto camera and $3 \mathrm{D}$ recovery for a forest scene in Amazon rain forest. The average height of the airplane is $H=385 \mathrm{~m}$, and the distance between the two slit windows is selected as 160 pixels (in the $y$ direction) with images of $720(x) * 480(y)$ pixels. The image resolution is about 7.65 pixels/meter. The depth map (Fig. 6c) generated from the stereo mosaics (Fig. 6 a and b) was obtained by using a hierarchical sub-pixel dense correlation method [16], where the range of depth variations of the forest scene (from a stereo fixation plane) is from $-24.0 \mathrm{~m}$ (tree canopy) to $24.0 \mathrm{~m}$ (the ground). Even before any 3D recovery, a human observer can perceive the $3 \mathrm{D}$ scene with a stereo pair using a pair of red/blue stereo glasses (Fig. 6d).

Multiple oblique parallel-perspective mosaics generated in a similar way can be used for image-based rendering as discussed in Section 4. A mosaic-based fly-through demo may be found at [17], which uses 9 oblique mosaics generated from a real video sequence of the UMass campus. This demo shows motion parallax, occlusion and moving objects in multiple parallel-perspective mosaics. We note that the rendering shows parallel-perspective rather than true perspective perception. A true perspective fly-through will be enabled by $3 \mathrm{D}$ reconstruction from the multiple mosaics.

\section{B. Video Mosaics for Under-Vehicle Inspection}

As one of the real applications of full parallel stereo mosaics, we have generated an approximate version of mosaics with full parallel projections from a virtual bed of 2D camera arrays by driving a car over a 1D array of cameras in an under-vehicle inspection system (UVIS) [13, 14, 18]. UVIS is a system designed for security checkpoints such as those at borders, embassies, large sporting events, etc. It is an example of generating mosaics from very short-range video so a $2 \mathrm{D}$ virtual array of camera is necessary for full coverage of the vehicle undercarriage.

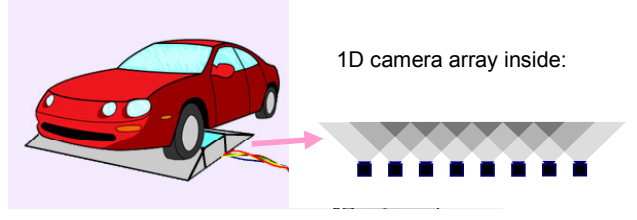

Fig. 7. 1D camera array for under-vehicle inspection $[13,14]$

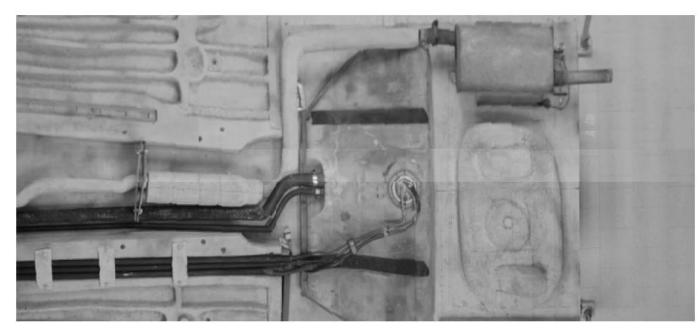

Fig. 8. Full parallel projection mosaics with a bed of 2D array of cameras

Fig. 7 illustrates the system setup where an array of cameras is housed in a platform. When a car drives over the platform, several mosaics with different oblique angles of the underside of a car are created. The mosaics can then be viewed by an inspector to thoroughly examine the underside of the vehicle from different angles. Fig. 8 shows such a mosaic generated from four overlapping image sequences taken by four moving cameras side by side simulating the motion of the vehicle. A PPT demo of five oblique parallel views of the mosaics can be found at [18] where different "occluded" regions under a pipe in the center can be observed by switching to different mosaics.

\section{CONCLUSIONS}

This paper presents an approach of fusing images from many video cameras or a moving video camera with external orientation data into a few mosaiced images with oblique parallel projections. In both cases, a virtual 2D array of cameras with FOV overlaps is formed to generate the whole coverage of a scene (or an object). The proposed representation provides wide FOV, preserves optimal 3D information, and represents occlusions. This representation can be used as both an advanced video interface for surveillance or a pre-processing step for 3D reconstruction.

\section{ACKNOWLEDGMENT}

This work is supported by NSF EIA- 9726401, AFRL Grants FA8650-05-1-1853 and F33615-03-1-63-83, and by funding from New York Institute for Advanced Studies and from Atlantic Coast Technologies, Inc.

\section{REFERENCES}

[1].J Y Zheng and S Tsuji, Panoramic representation for route recognition by a mobile robot, IJCV, 9(1), 1992: 55-76

[2]. S Peleg, B Rousso, A Rav-Akha, A. Zomet, Mosaicing on adaptive manifolds, PAMI, 22(10), Oct 2000: 1144-1154.

[3].USGS DEM, http://data.geocomm.com/dem/

[4]. S Peleg, M Ben-Ezra and Y Pritch, OmniStereo: panoramic stereo imaging, PAMI, March 2001:279-290.

[5].H-Y Shum and R Szeliski, Stereo reconstruction from multiperspective panoramas, ICCV'99: 14-21.

[6].S Baker, R Szeliski and P Anandan, A layered approach to stereo reconstruction. $C V P R^{\prime} 98$ : 434-441

[7].J Shade, S Gortler, L He. and R Szeliski, Layered depth image. SIGGRAPH'98: 231-242

[8].Z Zhu and A R Hanson, LAMP: 3D Layered, Adaptive-resolution and Multi-perspective Panorama - a New Scene Representation, CVIU 96(3), Dec 2004: 294-326.

[9].A Zomet, D Feldman, S Peleg, D Weinshall, Mosaicing new views: crossed-slits projection, PAMI 25(6), June 2003.

[10].J Chai and H-Y Shum, Parallel projections for stereo reconstruction, CVPR'00: II 493-500.

[11].Z Zhu, E M Riseman, A Hanson, Generalized Parallel-Perspective Stereo Mosaics from Airborne Videos, PAMI 26(2), Feb 2004:226-237.

[12].Z Zhu, A R Hanson, H Schultz and E M Riseman, Generation and error characteristics of parallel-perspective stereo mosaics from real video. In Video Registration, M. Shah and R. Kumar (Eds.), Kluwer, 2003: 72105.

[13].P Dickson, J Li, Z Zhu, A R Hanson, E M Riseman, H Sabrin, H Schultz and $\mathrm{G}$ Whitten, Mosaic generation for under-vehicle inspection. WACV'02:251-256

[14].http://vis-www.cs.umass.edu/projects/uvis/index.html

[15].http://www-cs.engr.ccny.cuny.edu/ zhu/StereoMosaic.html

[16].H Schultz, Terrain reconstruction from widely separated images, SPIE 2486, April 1995: 113-123.

[17].http://www-cs.engr.ccny.cuny.edu/ zhu/CampusVirtualFly.avi

[18].http://www-cs.engr.ccny.cuny.edu/ zhu/mosaic4uvis.html 